\title{
Steady-state security in distribution networks with large wind farms
}

\author{
Dilan JAYAWEERA ( $\square)$, Syed ISLAM
}

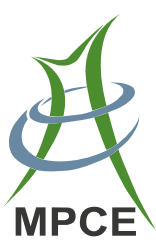

\begin{abstract}
Aging network assets, forced and unforced outages, and the way the networks are operated in a deregulated market are of significant concerns to integrate large wind farms in a distribution network. In many cases, the constrained network capacity is a potential barrier to the large-scale integration of wind power. This paper probabilistically assesses the steady-state security in a distribution network in the presence of large wind farms. The approach incorporates active distribution network operating conditions, including intermittent power outputs, random outages, demand fluctuations, and dynamic interactions and exchanges, and then assesses the steady state security using Monte Carlo simulation. A case study is performed by integrating large wind farms into a distribution network. The results suggest that intermittent outputs of large wind farms in a distribution network can impact the steady-state security considerably. However, the level of impact of wind farms does not necessarily correlate with the installed capacity of them.
\end{abstract}

Keywords Monte Carlo simulation, Network integration capacity, Random outages, Security of supply, Wind power generation

\section{Introduction}

Conventional distribution networks are less stressed compared to modern distribution networks because of

CrossCheck date: 12 April 2014

Received: 11 September 2013/Accepted: 18 March 2014/Published Online: 29 April 2014

(C) The Author(s) 2014. This article is published with open access at Springerlink.com

D. JAYAWEERA, S. ISLAM, Curtin University, Perth,

WA 6102, Australia

$(\bowtie)$ e-mail: dilan.jayaweera@ieee.org centrally generated power is aimed at transmitting through network assets to end-use customers. However, the usability of central generation also depends on the availability of in-service assets of a distribution network. Modern distribution networks are moving towards the active operation where the voltage and power flows are also controlled by distributed generation (DG). Among the $\mathrm{DG}$, the wind and photovoltaic (PV) generated electricity dominates the load share in most cases. Outputs of wind and PV are naturally intermittent and they can potentially impact the security of supply to customers.

Integration of wind and PV requires additional generation supports to mitigate impacts of intermittent effects. This additional generation can be supplied in the form of standby generation. On the other hand, energy storage solutions can also be used to mitigate impacts of intermittent effects. However, the economic competitiveness of large capacities of storage technologies against high penetration of wind power is not necessarily attractive. Intermittent effects can also produce intermittent stresses onto a distribution network, resulting in early retirement of assets.

With the paradigm shifts, the research problems of integrating wind generation into active distribution networks are explored in a wider spectrum. The DG penetration based on the harmonic limit, increase in DG penetration with the energy storage, protection coordination with the DG penetration, optimal allocation of DG with mathematical programming, capacity sufficiency with DG integrations, and reliability levels of active distribution networks are explored in [1-9]. The spinning reserve requirement for a significant penetration of wind power generation is explored in [10].

Power restoration practices of active distribution networks reached a new paradigm, and they are investigated in [11]. Some researchers developed new techniques to model wind power generation characteristics [12]. Security with the intermittent wind power is explored in [13]. 
Economic analysis of a DG mixture, long term simulation models of intermittent DG, and the dispersed generation and their correlation factors are investigated in [14-16]. A method is proposed in [17] to assess reliability of utility and industrial power generation systems. In [18], results from a customer survey are presented. Its findings suggest that customers with a backup system pay more per event than those without.

In recent years, the attention of the Plug-in Hybrid electric vehicles (PHEV) is increased as a mobile source of DG. They can also be used as a strategic source to mitigate stresses. In [19], adaptability of electric vehicle aggregation to electricity market is explored in the context of enhancing the security of power supply. In [20], the impact of PHEV behavior on the electric grid is analyzed by taking into account daily driving schedules. In [21], impacts of PHEV charging patterns on the power system operation are explored using stochastic unit commitment models. In [22], an approach is proposed to model PHEV home charging patterns, taking into account the stochastic nature of individual loads. In [23], a distributed framework for demand response and the user adaptation is explored in the context of smart grids, taking into account the pricing of the congestion. In [24], a three-state weather model is proposed for the reliability assessment. In [25], a technique is presented to reduce the errors in the short-term load forecasting. Reference [26] argues that number of transmission line outages is not necessarily proportional to the physical lengths of lines for many transmission systems. In [27], the value of security is quantified with inter-regional transmission lines. Security impacts with the large-scale integration of wind power are explored in [28]. In [29], a multi objective probabilistic risk index is proposed to capture the likelihood and consequences of events. Fuzzy and Monte Carlo simulation based hybrid technique is proposed in [30] for the assessment of power system risk. Multiobjective optimization based algorithm is presented in [31] for the active distribution network planning, taking into account uncertainties in DG and the demand response. Reference [32] argues that the statistical fitting techniques are not necessarily applicable to characterize the observed learning behavior.

The steady state security can be assessed deterministically as well as probabilistically. In a deterministic security assessment, the number of contingencies can be limited to $\mathrm{N}-1$ or N-2 criterion and each component of the distribution network is assumed to have an equal probability of failure or in other words no probabilistic terms is taken into account. In contrast, in a probabilistic assessment, the number of outages is determined by state random sampling.

This paper proposes a probabilistic approach to assess the steady-state security in an active distribution network in the presence of large wind farms. The approach quantifies the impact of intermittent outputs of large wind farms as a function of severity and the duration of impacts. The Monte Carlo simulation is the main engine of the probabilistic approach that incorporates the minimum loadshedding routines at various stages of remedial actions. The active distribution network operating conditions are modeled by incorporating random outages, intermittent power outputs, dynamic interactions and exchanges, and sector customer load demand fluctuations. The paper also extends the approach in the context of capacity credits with large wind farms.

The remaining sections of the paper are organized as follows. Section 2 presents the approach. It also describes the Monte Carlo simulation steps and the minimum load shedding formulations. Section 3 presents a case study and critically analyses the results. Section 4 presents conclusions.

\section{The approach}

There are many uncertainties in an active distribution network and most of them are stochastic in nature. Due to the level of uncertainties exists in an active distribution network, a probabilistic approach is necessary to capture the rational characteristics of large wind farms that can impact the steady-state security. The approach proposed in this paper incorporates non-sequential Monte Carlo simulation to capture uncertainties of operating conditions of an active distribution network [27].

In a power distribution network, the network assets can experience stresses as a consequence of disturbances. The approach classifies the stresses into two types. The first type is the stress that arises from outages and other events excluding intermittent effects of DG and dynamic interactions. The second type is the stress that can occur due to intermittent effects of DG outputs and the dynamic interactions of stochastic power generating units with conventional units such as diesels.

The severity of disturbances in a network that leads to the first and the second types of stresses can be quantified using different metrics. One of the metric that can be used to quantify the stress is the level of shed-load. This is because if a network experiences a stress that cannot be eliminated without shedding loads, the ultimate decision of a network operator is to initiate the load shedding. The constraint violation in a network can also be considered as an early indication of a system that heads towards a stressed operating condition, however, it is a tentative indication of stress because not all constraint violations lead to load shedding. 
The shed load does not completely reflect the system stress because the system stress should also capture the electricity customers' stress, which is the product of the magnitude of the shed-load and the time to restore the shed-load. Thus, the approach considering the restoration time of the shed-load is also as a part of the system stress quantification metric.

In addition, in a power transmission network, the shedload can be restored using a step-by-step restoration scheme that uses different restoration rates (MW/minute) or a continuous restoration scheme that uses a single restoration rate. Typically, most power transmission network loads are aggregated loads that represent distribution networks. The security standards of power transmission systems recommend the rate of restoration of a shed-load and the time threshold to restore the curtailed load. However, in distribution networks, the shed-load can be restored using a single rate of restoration scheme because of the loads that are to be restored are considerably lower in magnitude compared with power transmission networks. Thus, in a trial in Monte Carlo simulation, a single rate of restoration can be applied to calculate the restoration time of shed loads of a network. However, multiple restoration rates can also be applied as per the requirement of a network.

A network can experience stresses when the intermittency of wind power generation coincides with critical operating conditions. Therefore, the shed-loads resulting through all disturbances are referred in this paper as the total shed-load. The total shed-load can be categorized into four elements of shed-loads. They are the shed-load due to inadequacy of wind power generation (excluding dynamic interactions and exchanges resulting shed-load), dynamic interactions and exchanges, contingencies, and unavailability of standby power generation. A detailed description of the dynamic interactions and exchanges is given in section 2.2. The first two shed-load components are influenced by intermittency of wind power generation. Therefore, the term 'shed-load due to intermittency of wind power' refers to the sum of the first two elements of shed-loads.

The second shed-load type arises as a consequence of interaction of intermittency of wind power with other disturbances. The sign of the second element can be either positive or negative as it depends on the network operating condition and the criticality of the post-disturbance state. Some of the post disturbance operating conditions are less stressed and can also lead to reduce the level of shed-loads.

\subsection{Modeling load demand and wind power output}

The load demand and intermittency of wind power output can be characterized by using time series of their outputs. In order to reduce the processing time of Monte Carlo simulation, the approach incorporates characteristic

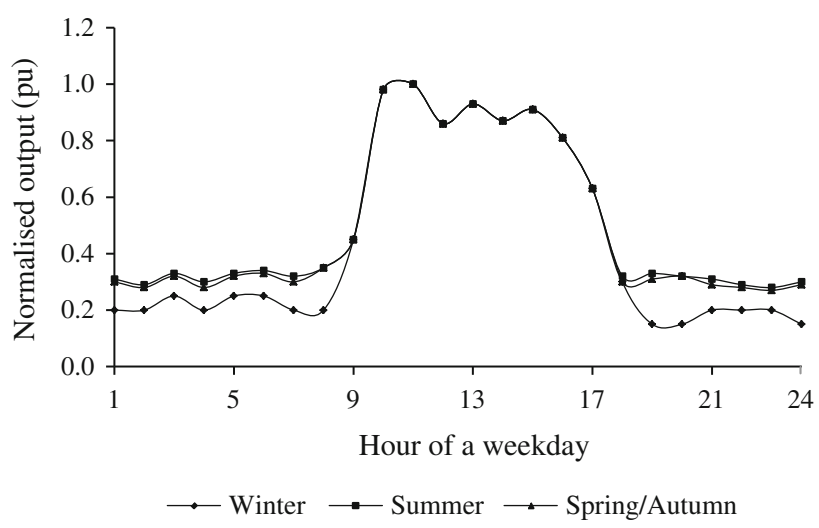

Fig. 1 An example of weekday's load demand characteristic profile of an industrial customer

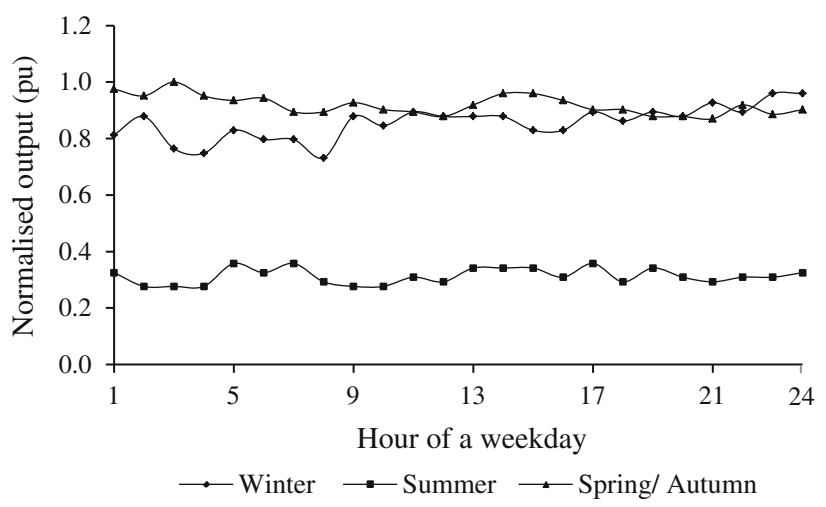

Fig. 2 An example of weekday's generation characteristic profile of an onshore wind farm

profiles of the load demand and the wind-power outputs. Incorporation of characteristic profiles does not compromise the accuracy of the estimation because the zero wind power generation states are captured through random state sampling of wind units (as it does in conventional unit outage modeling) and other generation states are modeled through characteristic profiles. This limits the averaging effects of characteristic profiles. The conventional unit outage modeling process is described in section 2.2.

Characteristic profiles are calculated by truncating yearly profiles into characteristic days. Days in each week are categorized into characteristic Weekday, Saturday, and Sunday. Seasons in each year are categorized into characteristic winter, summer, and spring/autumn. Thus, each season can produce characteristic Weekday, Saturday, and Sunday and in total the process produces nine types of days in a year. In other words, the process produces 216 samplehours to represent a characteristic profile. Figures 1 and 2 show weekday's characteristic profiles of an industrial type customer and onshore wind farm respectively. Both profiles are derived from yearly profiles. 
On the other hand, the entire time series of wind and the sector customer load demand can also be incorporated into Monte Carlo simulation without truncating but reducing the variance of the estimation by applying a variance reduction technique.

\subsection{Monte Carlo simulation}

Monte Carlo simulation incorporates a healthy network model to begin the simulation. The healthy network model is used as the base case and the model should be free from voltage limit and thermal limit violations. On top of the basecase, random outages are modeled. Random outages are modeled by using random sampling of states where uniformly distributed random numbers are generated between 0 and 1.0 for each network component and then they are compared with the probability of the outage of each component. If the generated random number for the equipment is less than the probability of the outage of the equipment then the equipment status is set as out of service. The probabilities of outage of equipment are calculated using statistical data of component failure rates. Following the convergence of Monte Carlo simulation, the failure rates generated by random numbers are almost equal to the statistical failure rates of equipment. Occurrences of random outages can change the network configuration which leads to re-assess the power balance using A/C power flow solution. Loads are shedded if the operating condition leads to the divergence of the power flow solution [27, 33].

Some of the bus and branch outages may trip neighboring branches. Similar to the components outage modeling, the protective relay actions are modeled by using random sampling of relay operations. This requires the statistics of tripping of neighboring branches. Clearing a fault can initiate dynamic interactions and exchanges at some operating conditions. These interactions mostly occur due to the existence of large wind turbine generators because the rapid responsive generators respond faster than slow units (e.g. wind). The plants that interact dynamically share the network load in an oscillatory pattern due to the constant low frequency power angle oscillations interacting in the system, which can also continue for an extended period of time. Depending on time constants of units and their controls, the resulting operating condition may even destabilize weak units and disconnect them from the network. The instability tripping of units due to dynamic interactions are also modeled by using random sampling of stability of units.

On the other hand, performing the transient stability analysis in parallel with Monte Carlo trials can also extract the units that are to be disconnected due to the dynamic interactions and exchanges. Similar philosophy can be applied for the protection relay operation to determine the tripping of neighboring branches. These options reduce the necessity of random sampling and the outcome can directly be applied to the sample trial of Monte Carlo simulation. However, the parallel processing of the entire transient processes can demand a significantly large processing time. Such issues can be limited by applying a probabilistic approach.

Next, the resulting operating condition is re-assessed to establish the power balance. Any divergence condition is rectified by applying the load shedding routine. The mathematical framework to shed the minimum level of loads is given through (1) to (6).

Minimize

$$
\begin{aligned}
& \sum_{k \in \text { ng-flex }} C_{k} P_{k}^{\text {gen-flex }}+\sum_{k \in \text { ng-nonflex }} C_{k} P_{k}^{\text {gen-nonflex }} \\
& \quad+\sum_{k \in \text { nld }} W_{k} C_{k}^{\text {shed }} P_{k}^{\text {shed }}
\end{aligned}
$$

Subject to:

Power flow through a branch,

$$
\begin{aligned}
T_{j} & =\sum_{k=1}^{n b} A_{k}^{j}\left(P_{k}^{\text {gen-flex }}+P_{k}^{\text {gen-nonflex }}-P_{k}^{\text {load }}+P_{k}^{\text {shed }}\right) ; \\
& (j=1, \ldots, n b r)
\end{aligned}
$$

Power balance at a bus,

$$
\begin{aligned}
& \sum_{k \in \text { ng-flex }} P_{k}^{\text {gen-flex }}+\sum_{k \in \text { g-nonflex }} P_{k}^{\text {gen-nonflex }}+\sum_{k \in \text { nld }} P_{k}^{\text {shed }} \\
& \quad=\sum_{k \in \text { nld }} P_{k}^{\text {load }}
\end{aligned}
$$

Generation limit constraint,

$P_{k}^{g e n-f l e x_{\min }} \leq P_{k}^{\text {gen-flex }} \leq P_{k}^{\text {gen-fle }}$ max $; \quad(k \in n g)$

Load shedding constraint,

$0 \leq P_{k}^{\text {shed }} \leq P_{k}^{\text {load }} ; \quad(k \in$ nld $)$

Branch flow constraint,

$\left|T_{j}\right| \leq T_{j}^{\max } ; \quad(j=1, \ldots, n b r)$

where $P_{k}^{\text {gen-flex }}, P_{k}^{\text {gen-nonflex }}, P_{k}^{\text {load }}$ and $P_{k}^{\text {shed }}$ are the flexible generation (dispatchable), non-flexible (including intermittent DG), load, and load shedding at the $k^{\text {th }}$ bus respectively. $A_{k}^{j}$ is the elements of the connectivity matrix between branch flows and power injections for the trial. $T_{j}$ is the power flow through the $j^{\text {th }}$ branch. $P_{k}^{\text {gen-flex }}$ min and $P_{k}^{\text {gen-flex }} x_{\max }$ are the lower and upper capacity limits of generation at the $k^{\text {th }}$ bus respectively. $T_{j}^{\text {max }}, W_{k}, C_{k}^{\text {shed }}$ and $C_{k}$ are the thermal limit of the $j^{\text {th }}$ branch, weighting factor that reflects the importance of the $k^{\text {th }}$ bus for load shedding, cost of the shed load factor at bus $k$, and unit generation cost for the $k^{\text {th }}$ bus generation respectively. nld, $n g-f l e x, n g-n o n f l e x, n b$ and $n b r$ are the total number of load buses, flexible generator buses, non-flexible generator, buses, and branches in the network respectively. 
Wind plants have two dispatch options: use the entire power output of plants of the trial or curtail the output if that is feasible. Curtailment of output of wind plants is possible if there are a number of wind turbine generators that share a network demand. If there is a single wind turbine generator, then it is disconnected from the network because of the practical limitations of curtailing outputs. However, wind plants can also be considered as limitedflexible plants if the alternative platforms are available to control the outputs of wind plants entering the power grid. These alternative platforms include energy storage solutions, micro grids, and flexible controls that can adjust the active power output of a wind farm.

The solution of the linear programming problem is accepted as a feasible solution if the operating condition of the network relating to the solution is within voltage and frequency statutory limits. The compliance is checked before leaving the load shedding routine of a trial. If the network achieved a stable operating condition, then the calculated results should indicate the existence of any thermal or voltage limit violations. The thermal limit violations are corrected by reconfiguring the network and redispatching generation by taking into account the merits and the flexible order of units. Then, on load tap changing and shunt compensation actions are applied if they are required to be deployed. At last, the loads can be shedded if the operating condition of the network is critical.

A set of other actions are also modeled to control over and under voltage conditions of the network. The over-voltage conditions are mitigated by switching-in shunt reactors, taking off switchable capacitor banks, reducing taps of connected transformers, and at last opening the lightly loaded lines if they are relevant. Under voltage conditions are controlled by switching shunt reactors off, switching capacitor banks in, switching line reactors off, tap operation of transformers, and at last loads are shedded only if the condition is critical.

Shed-load due to unavailability of standby power is calculated by following the steps described earlier but excluding the outages and dynamic interactions and exchanges of standby power units. The entire process described above occurs in a sample trial in Monte Carlo simulation. At the end of each trial, ENS (energy not supplied) for the trial is calculated and EENS (expected ENS) for the completed trials and impact factor on steady-state security due to large wind farms are estimated. Calculation of ENS, EENS, and impact factor due to large wind farms are described in 2.3 to 2.5.

Monte Carlo simulation performs a number of trials to estimate the shed-load components and to satisfy the convergence criteria. It stops if the expected degree of confidence of the shed-load is within the confidence limit and the number of trials being processed is within the range of the minimum and maximum trials. It can also be stopped if the simulation reaches the maximum number of trials

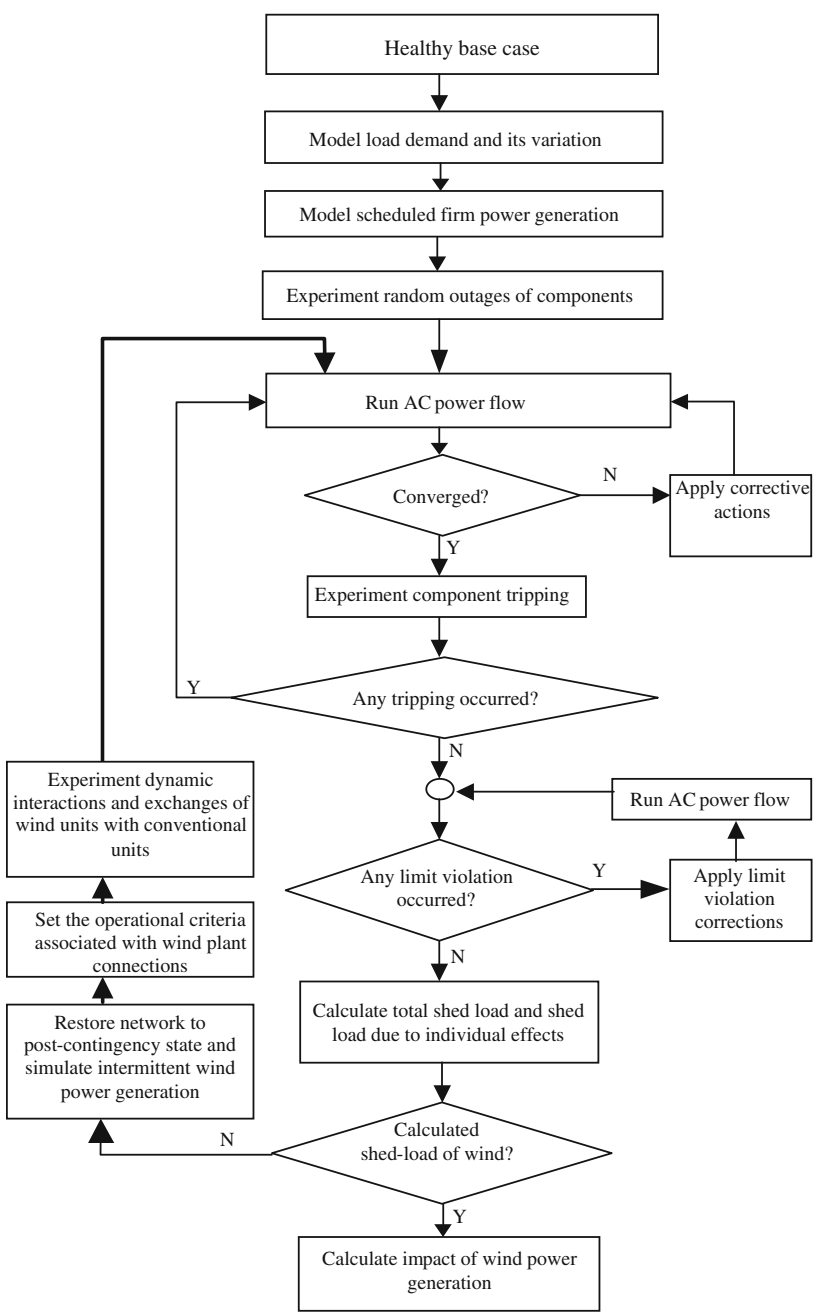

Fig. 3 A sample trial calculation of impact of wind power on steadystate security

without satisfying the degree of confidence and confidence interval. In that case, the estimated value does not have an acceptable degree of confidence and is inconclusive. Figure 3 shows the basic steps of calculating the impact of wind power on the steady-state security in a sample trial in Monte Carlo simulation, where 'corrective action' referred to applying load-shedding routine described above and the 'limit violation correction' referred to correcting the constraint violation using the actions described above.

\subsection{Calculation of ENS}

The energy not supplied is defined as the product of the magnitude of the shed-load and the time to restore the shed-load. The total energy not supplied in a trial of Monte Carlo Simulation is mainly due to the intermittency of generation output and outages. These two types of events are not linearly associated with the total ENS due to the fact that the restoration schemes operate based on the total 
magnitude of the shed-load. However, the total shed-load is linearly associated with the shed-load due to intermittent effects and outages, and they can be weighted in accordance with their contributions. Therefore, the weighting factors are calculated with the shed-load, and then they are incorporated into the calculation of ENS. Thus, the ENS due to intermittency of wind power generation at a trial is calculated by using

$E N S_{\mathrm{int}}^{i}=\frac{\left(P_{\text {shed }_{\mathrm{int}}}^{i} \times E N S_{\text {tot }}^{i}\right)}{P_{\text {shed }_{\text {tot }}}^{i}}$

where $E N S_{\text {tot }}^{i}$ is the total ENS at a trial $i$ and calculated by using

$E N S_{\text {tot }}^{i}=\sum_{k=1}^{m} P_{\text {shed } d_{t o t}}^{k} \times T_{\text {rest }}^{k}$

where $P_{\text {shed }}^{k}$ is the total shed-load that is restored during the time $T_{\text {rest }}^{k}$, and $m$ is the number of shed-load components that form the total shed-load. Shed-load due to intermittency $\left(P_{\text {shed }}^{i}\right)$ is the difference between the total shed-load $\left(P_{\text {shed }}^{i}{ }_{\text {tot }}\right)$ and the shed-load due to contingencies

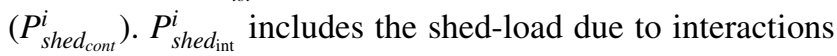
of wind plant dynamics with other disturbances. $P_{\text {shed }}^{i}$ excludes interactions of the shed-load of wind plant dynamics with other disturbances. $P_{\text {shed }}^{i}$ is is due to all disturbances during the trial $i$.

\subsection{Estimation of ENS (EENS)}

The energy not supplied due to intermittency of wind power generation and dynamic interactions and exchanges is estimated by using

$E E N S_{\text {int }}=\frac{1}{n} \sum_{i=1}^{n}\left(P_{\text {shed }_{\text {int }}}^{i} \times E N S_{\text {tot }}^{i}\right) / P_{\text {shed }_{\text {tot }}}^{i}$

where $P_{\text {shed }}^{i}$ is the shed due to the intermittency of windpower output and dynamic interactions and exchanges at the trial $i$, and $n$ is the number of Monte Carlo trials being processed. The EENS due to the unavailability of the standby power generation to eliminate intermittent effects is estimated by using (10)

$E_{E N S_{s t d b}}=\frac{1}{n} \sum_{i=1}^{n}\left(P_{\text {shed }_{\text {std }}}^{i} \times E N S_{\text {tot }}^{i}\right) / P_{\text {shed }_{\text {tot }}}^{i}$

where $P_{\text {shed }_{\text {std }}}$ is the shed-load that is purely due to the inadequacy of the wind-power generation to supply the demand at the trial $i$. On the other hand, it can also be defined as the required standby power generation to preserve the firm power generation capacity with wind plants.
2.5 Impacts of large wind farms

The impact of large wind farms on steady-state security is calculated by increasing the installed capacities of wind farms gradually (from zero to feasible limits of installed capacities) and then estimating the ENS at each of the increments. The impact factor of large wind farms on steady-state security $(\mu)$ can be calculated by using (11)

$\mu=\frac{E E N S_{\text {int }}}{E E N S_{\text {tot }}} ; \quad 0 \leq \mu \leq 1.0$

where $E E N S_{\text {tot }}$ is estimated by using

$E_{E N S_{\text {tot }}}=\frac{1}{n} \sum_{i=1}^{n} E N S_{\text {tot }}^{i}$

The factor $(\mu)$ gives the impact of wind generated electricity on the steady state security with the presence of large wind farms in a scale of zero to one. For the central connection of wind farms, the lowest impact factor that provides the largest installed capacity is the maximum capacity that can be integrated into the network safely because it gives the least impact on the steady-state security yet absorbing a relatively larger installed capacity of wind. On the other hand, a medium level impact factor with a medium level installed capacity of wind can also be benefitted for a network. For the decentralized connection of wind farms, the same rule can be applied, however, the impact factor due to large wind farms can be lower compared to central integration because of the ability of decentralized connections of wind farms to provide firm power outputs with auxiliary resources.

\subsection{Generation capacity credits}

The wind capacity credits can be seen through the proposed impact factor of large wind farms. In order to receive capacity credits with the integration of large wind farms, there should be more than one wind plant site that shares the demand of the network. This is because the combination of all wind farm outputs makes the minimum capacity, which enables to maintain the same or a better security of supply compared with the case without wind plants. Therefore, the total installed capacity that provides the largest firm capacity with a smallest impact factor can be considered as the capacity credit that does not compromise the security of supply to customers.

\section{Case study}

The aim of the case study is to demonstrate the performance of the proposed approach and to probabilistically assess the wind integration capacity that provides the lowest impact on the steady-state security in an active distribution network. Figure 4 shows a single-line diagram 


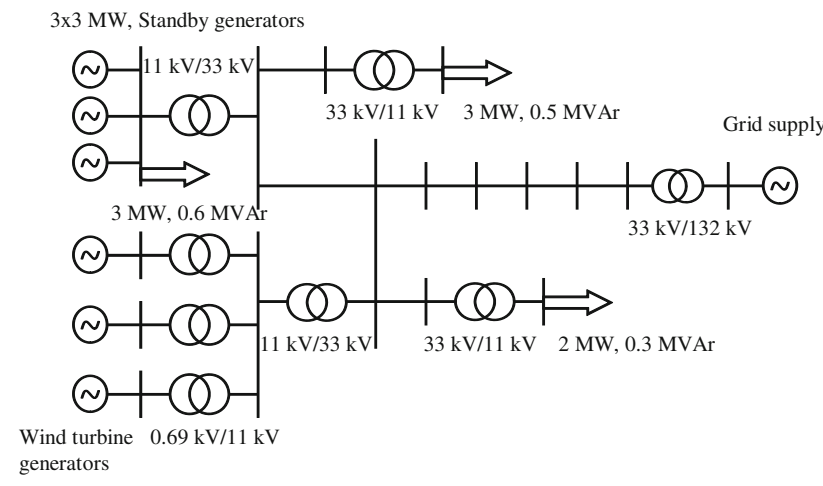

Fig. 4 Single line diagram of a rural distribution network

of a rural distribution network that facilitates the connection of wind plants. The network has 17 buses and eight transformers of which the 15 MVA $33 / 132 \mathrm{kV}$ transformer is a voltage regulating transformer with on-load tap changing facility.

The generating capacity of the standby generating plant is $9 \mathrm{MW}$. It is connected to the $33 \mathrm{kV}$ grid via a $10 \mathrm{MVA}$ $11 \mathrm{kV} / 33 \mathrm{kV}$ transformer. There are three wind plants, which have similar operating characteristics. Wind plants operate at the unity power factor. The load demand of the network is shared by wind/standby or wind/grid generating unit combinations.

Four types of customers are connected at each load bus. They are residential restricted, residential unrestricted, industrial, and commercial. The peak active and reactive power loads on the network are $8 \mathrm{MW}$ and 1.4 MVAr respectively. The loads are susceptible to fluctuations.

The network load and the wind farm capacity factors are 0.48 and 0.28 respectively. The minimum and the maximum number of trials of Monte Carlo simulation were set as 8,000 and 100,000 respectively. Monte Carlo simulation stops if it meets the stopping criteria detailed in section 2 . Truncated characteristic profiles of loads and wind power generation are applied during the simulation study.

\subsection{Scenarios}

The scenarios are built upon a case where the grid shown in Fig. 4 is planning to supply an increase in load demand that requires upgrading the grid. The need for upgrading the grid can be differed by increasing the wind generation capacity at the site shown in Fig. 4. However, the impact of large wind farms on steady state security is to be estimated in order to justify the case. The proposal is accepted if the impact on steady state security does not compromise the existing level of security. It is also required to assess the installed capacity of wind units that meets the said requirement.

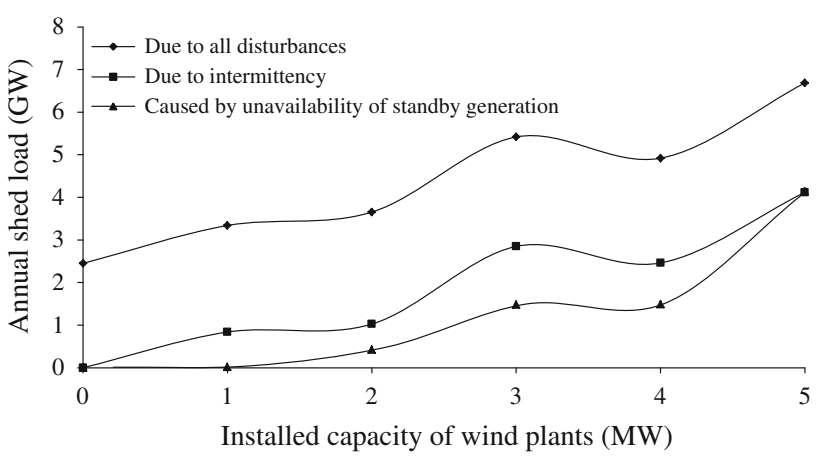

Fig. 5 Annual shed-load due to disturbances

Six scenarios were designed to assess the viability of the proposal. The first scenario represents the base case which has no wind plants connected and is used to compare merits of other scenarios. The second through six scenarios have installed capacities of wind units from $1 \mathrm{MW}$ to $5 \mathrm{MW}$ in $1 \mathrm{MW}$ steps respectively.

The methodology proposed in section 2 is applied to each scenario. A trial of the Monte Carlo simulation represents an hour-long duration. The expected certainty and confidence limit are set as $95 \%$ and $5 \%$ respectively. Thus, the simulation results should have a $95 \%$ confidence of the estimated mean (shed-load) within $5 \%$ of the true mean for conclusive arguments.

\subsection{Results}

Each scenario lasted a time between 1 to 2 hours to converge. Figures 5, 6 and 7 show the annual shed-load, annual EENS, and impact factor $(\mu)$ for the increased installed capacities of wind units. Annual shed-load and annual EENS almost follow a similar trend because of the level of loads to restore in a distribution network is relatively lower compared to power transmission systems and most of the restorations can be done with a single step of restoration. There are exceptional cases, which are not considered for the studies.

\subsection{Analysis}

The key message of the results is that the steady-state security impact of wind power in an active distribution network is non-linear and a quantitative assessment is needed for assessing true impacts. The impact factor proposed in the paper has the ability to signal about the accommodation capacity that provides the lowest stress to a network. Increase in installed capacity of a wind power generating unit does not necessarily increase the system stress. A network can have a threshold capacity of wind power beyond which the impact factor of wind can be increased significantly. 


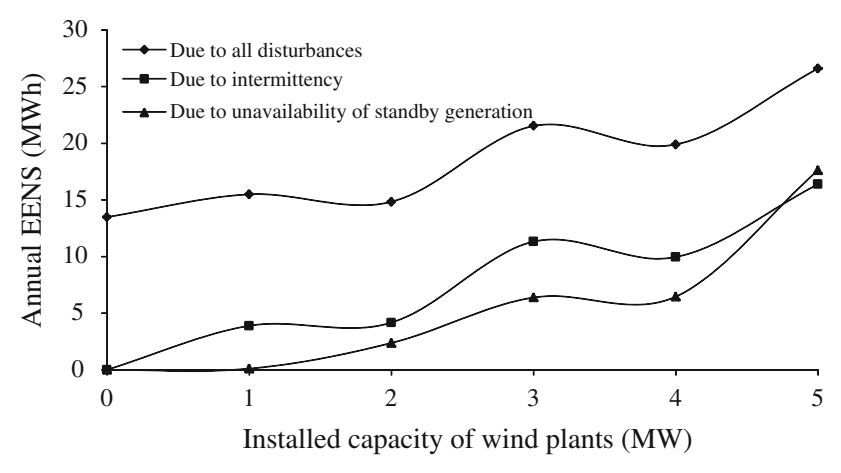

Fig. 6 Annual EENS due to disturbances

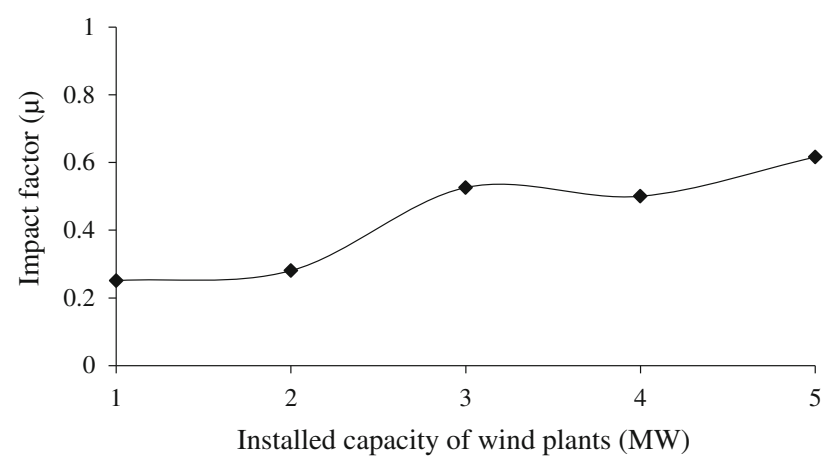

Fig. 7 Impact factor of large wind farms

Results in Figs. 5 and 6 argue that worth of using standby power generation increases with the increase in installation capacity of a wind unit. In this particular network, the contingency contribution to shed-load is approximately consistent with the increased installed capacity. Results further suggest that the increase in wind installed capacity does not necessarily increase the impacts of the intermittency of wind power. For example, installation of $2 \mathrm{MW}$ wind plant provides more benefit than the installation of $1 \mathrm{MW}$. However, if the standby generation is not available in the system then, the system can absorb a $1 \mathrm{MW}$ capacity than $2 \mathrm{MW}$. The fifth scenario (4 MW installed capacity) releases a better network capacity to accommodate wind compared to the fourth scenario (3 MW installed capacity) because of its reduced impact on the steady-state security. The reason for this occurrence is that the generation constraint at the fourth scenario limits supplying the full energy demand at some sample trials compared to the fifth scenario, and some of the post-fault operating conditions in the fifth scenario are also less stressed.

The impact factor in Fig. 7 suggests that integrating $2 \mathrm{MW}$ of wind capacity at the site delivers most benefits compared to any other case because it can limit the stress of intermittent wind power output as in $1 \mathrm{MW}$ case and helps reducing the need for fossil-fuelled power generation. Similarly, installation of $3 \mathrm{MW}$ and $4 \mathrm{MW}$ of wind provides similar stresses. However, they carry a higher level of impact compared to the impact of the scenario of $2 \mathrm{MW}$ installed capacity.

The steady-state impact of wind power output remains consistent for $1 \mathrm{MW}$ to $2 \mathrm{MW}$ of installed capacities of wind. However, the impacts considerably increase when the penetration level exceeds said level. Then, the impact marginally reduces at the $4 \mathrm{MW}$ of installed capacity of wind and thereafter it continues to increase. Such a variation occurs due to the severity of intermittency with the increased penetration of wind power and associated constraints of assets in the network.

The study results also affirm on the potential ability of the approach to quantify the steady-state security with the integration of large wind farms in an active distribution network. As an auxiliary outcome, the approach also produces the latent capacity of a network that delivers the lowest impact.

With the increased trend in modernizing traditional distribution networks towards smart distribution networks, the shares of wind and other intermittent generation technologies to supply the load demands can be increased considerably. In that context, the quantification of steadystate stresses due to wind power outputs can potentially provide vital information for network operators to take actions against uncontrollable conditions of a network. Such actions can also limit the interruption of power supply to electricity consumers.

\section{Conclusions}

The paper presents a Monte Carlo simulation based probabilistic approach to assess the steady-state security in an active distribution network. The approach quantifies the stresses caused by integration of large wind farms against other events.

Case study suggests that impacts of large wind farms on steady state security are inconsistent and a quantitative assessment can suggest the level of inconsistency.

Availability of standby power can potentially mitigate the impacts of intermittency. However, increase in wind power penetration does not necessarily increase the need for standby power. The effectiveness of standby-power to mitigate security impacts can be significant in high windpower penetrated distribution networks.

The approach can be applied to benchmark active distribution networks based on impacts on steady-state security with large wind farms. 
Open Access This article is distributed under the terms of the Creative Commons Attribution License which permits any use, distribution, and reproduction in any medium, provided the original author(s) and the source are credited.

\section{References}

[1] Bhowmik A, Maitra A, Halpin SM et al (2003) Determination of allowable penetration levels of distributed generation resources based on harmonic limit considerations. IEEE Trans Power Deliv 18(2):619-624

[2] Brahm SM, Girgis AA (2004) Development of adaptive protection scheme for distribution system with high penetration of distributed generation. IEEE Trans Power Deliv 19(1):56-63

[3] Chilvers I, Jenkins N, Crossley P (2005) Distance relaying of $11 \mathrm{kV}$ circuits to increase the installed capacity of distributed generation. IEE P-Gener Trans Dis 152(1):40-46

[4] Keane A, O'Malley M (2005) Optimal allocation of embedded generation on distribution networks. IEEE Trans Power Syst 20(3):1640-1646

[5] Billinton R, Bai G (2004) Generating capacity adequacy associated with wind energy. IEEE Trans Energy Convers 19(3): 641-646

[6] Chiradeja P, Ramakumar R (2004) An approach to quantify the technical benefits of distributed generation. IEEE Trans Energy Convers 19(4):764-773

[7] Barton JP, Infield DG (2004) Energy storage and its use with intermittent renewable energy. IEEE Trans Energ Conver 19(2): 441-448

[8] Karki R, Billinton R (2004) Cost-effective wind energy utilization for reliable power supply. IEEE Trans Energy Convers 19(2):435-440

[9] Quezada VHM, Abbad JR, Roman TGS (2006) Assessment of energy distribution losses for increasing penetration of distributed generation. IEEE Trans Power Syst 21(2):533-540

[10] Ortega-Vazquez MA, Kirschen DS (2009) Estimating the spinning reserve requirements in systems with significant wind power generation penetration. IEEE Trans Power Syst 24(1):114-124

[11] Pham TTH, Besanger Y, Hadjsaid N (2009) New challenges in power system restoration with large scale of dispersed generation insertion. IEEE Trans Power Syst 24(1):398-406

[12] Vallee F, Lobry J, Deblecker O (2008) System reliability assessment method for wind power integration. IEEE Trans Power Syst 23(3):1288-1297

[13] Wang J, Shahidehpour M, Li Z (2008) Security-constrained unit commitment with volatile wind power generation. IEEE Trans Power Syst 23(3):1319-1327

[14] Brown PD, Peas Lopes JA, Matos MA (2008) Optimization of pumped storage capacity in an isolated power system with large renewable penetration. IEEE Trans Power Syst 23(2):523-531

[15] Banakar H, Luo C, Ooi BT (2008) Impacts of wind power minute-to-minute variations on power system operation. IEEE Trans Power Syst 23(1):150-160

[16] Vallee F, Lobry J, Deblecker O (2007) Impact of the wind geographical correlation level for reliability studies. IEEE Trans Power Syst 22(4):2232-2239

[17] Chowdhury AA, Koval DO (2005) Impact of photovoltaic power sources on a power system's capacity reliability Levels. In: Proceedings of the 2005 IEEE industrial and commercial power systems technical conference (I\&CPS’05), Saratoga Springs, 8-12 May 2005, pp 32-37

[18] Chowdhury AA, Mielnik TC, Lawton LE et al (2009) System reliability worth assessment using the customer survey approach. IEEE Trans Ind Appl 45(1):317-322
[19] Ortega-Vazquez MA, Bouffard F, Silva V (2013) Electric vehicle aggregator/system operator coordination for charging scheduling and services procurement. IEEE Trans Power Syst 28(2):1806-1815

[20] Lee T, Adornato B, Filipi ZS (2011) Synthesis of real-world driving cycles and their use for estimating PHEV energy consumption and charging opportunities: case study for midwest/ US. IEEE Trans Veh Technol 60(9):4153-4163

[21] Liu C, Wang J, Botterud A et al (2012) Assessment of impacts of PHEV charging patterns on wind-thermal scheduling by stochastic unit commitment. IEEE Trans Smart Grid 3(2):675-683

[22] Grahn P, Munkhammar J, Widen J et al (2013) PHEV homecharging model based on residential activity patterns. IEEE Trans Power Syst 28(3):2507-2515

[23] Fan Z (2012) A distributed demand response algorithm and its application to PHEV charging in smart grids. IEEE Trans Smart Grid 3(3): 1280-1290

[24] Billinton R, Singh G (2006) Application of adverse and extreme adverse weather: modelling in Transmission and distribution system reliability evaluation. IEE P-Gener Trans Distrib 153(1): $115-120$

[25] Fay D, Ringwood JV (2010) On the influence of weather forecast errors in short-term load forecasting models. IEEE Trans Power Syst 25(3):1751-1758

[26] Shen S, Koval D, Shen S (1999) Modelling extreme-weatherrelated transmission line outages. In: Proceedings of the 1999 IEEE Canadian conference on electrical and computer engineering (CCECE'99), vol 3. Edmonton, 9-12 May 1999, pp 1271-1276

[27] Rios MA, Kirschen DS, Jayaweera D et al (2002) Value of security: modeling time-dependent phenomena and weather conditions. IEEE Trans Power Syst 17(3):543-548

[28] Grijalva S, Dahman SR, Patten KJ et al (2007) Large-scale integration of wind generation including network temporal security analysis. IEEE Trans Energy Convers 22(1):181-188

[29] Xiao F, McCalley JD (2009) Power system risk assessment and control in a multiobjective framework. IEEE Trans Power Syst 24(1):78-85

[30] Li W, Zhou J (2009) Power system risk assessment using a hybrid method of fuzzy set and Monte Carlo simulation. In: Proceedings of the IEEE Power and Energy Society general meeting (PES'09), Calgary, 26-30 July 2009, p 1

[31] Martins VF, Borges CLT (2011) Active distribution network integrated planning incorporating distributed generation and load response uncertainties. IEEE Trans Power Syst 26(4): 2164-2172

[32] Duffey RB, Ha T (2013) The probability and timing of power system restoration. IEEE Trans Power Syst 28(1):3-9

[33] Cochran WG (1977) Sampling techniques, 3rd edn. Wiley, New York

Dilan JAYAWEERA received the M.Sc. and Ph.D. degrees in electrical power engineering from the University of Manchester Institute of Science and Technology (UMIST), Manchester, U.K., in 2000 and 2003, respectively. He was with Imperial College, London and University of Strathclyde, Glasgow. Currently he works at Curtin University, Australia as a Senior Lecturer. He is a senior member of the IEEE and a chartered engineer in the United Kingdom.

Syed ISLAM received the M.Sc. and PhD degree in electrical power engineering from the King Fahd University of Petroleum and Minerals, Saudi Arabia, in 1983, and 1988 respectively. $\mathrm{He}$ is a Professor at Curtin University. He is a Fellow of Engineers Australia, Fellow of IET, and a chartered engineer in the United Kingdom. 